\title{
CORRIGENDUM
}

\section{CMC-544 (inotuzumab ozogamicin), an anti-CD22 immuno-conjugate of calicheamicin, alters the levels of target molecules of malignant B-cells}

A Takeshita, N Yamakage, K Shinjo, T Ono, I Hirano, S Nakamura, K Shigeno, T Tobita, M Maekawa, H Kiyoi, T Naoe, K Ohnishi, Y Sugimoto and R Ohno

Leukemia (2009) 23, 1372; doi:10.1038/leu.2009.115

Correction to: Leukemia (2009) 23, 1329-1336; doi:10.1038/ leukemia.2009.77; published online 16 April 2009
Since the publication of this paper, the authors have noticed an error in Figure 1. The correct figure is shown here.

The authors apologize for this error.
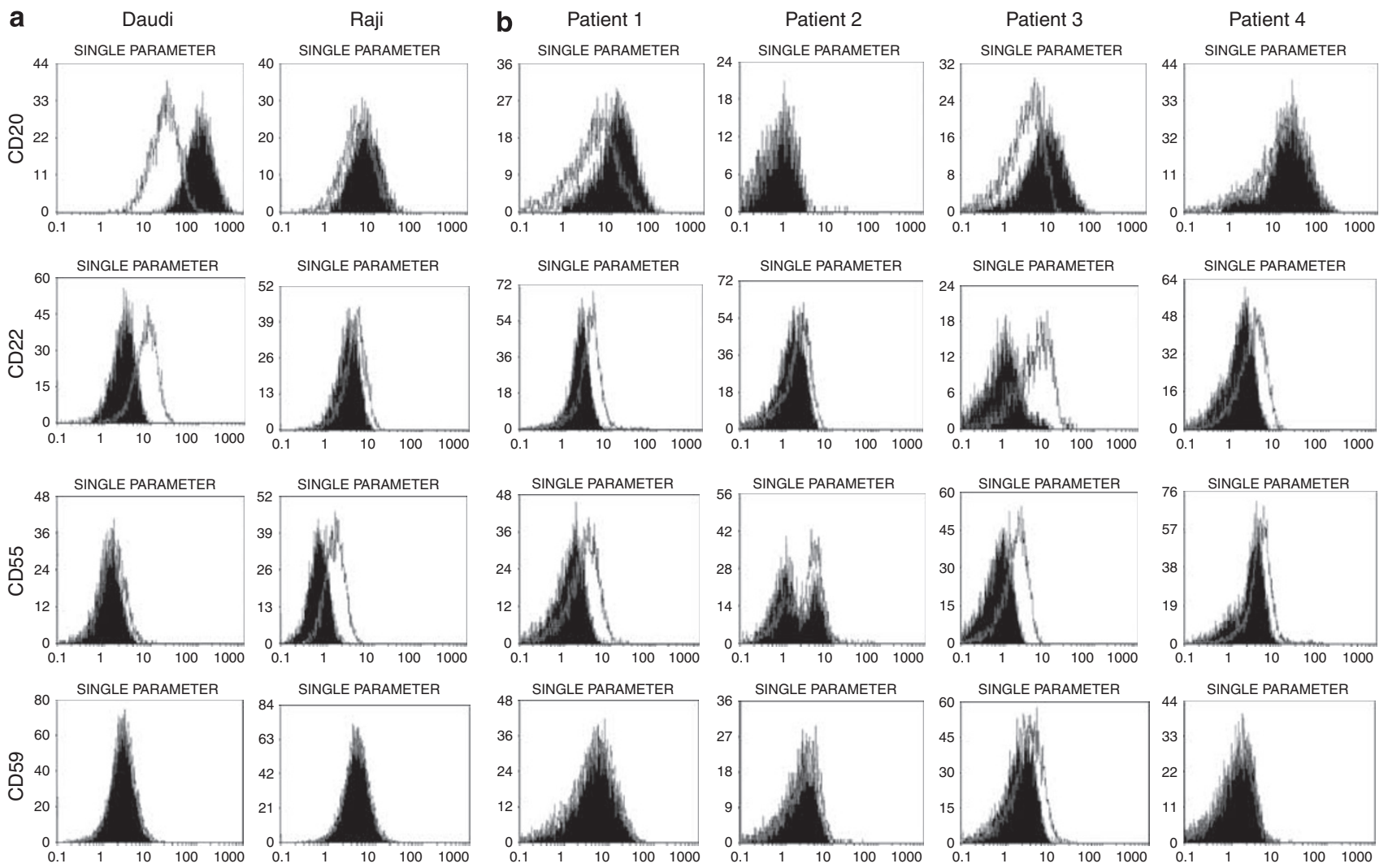

Figure 1 (a) The levels of CD20, CD22, CD55 and CD59 antigens on Daudi and Raji cells were analyzed by flow cytometry after exposure to a medium with or without CMC-544. The horizontal lines show the fluorescence intensity, and the vertical lines show the levels of CD20, CD22, CD55 or CD59. White and black histograms show the data obtained after cells were incubated with G5/44 or CMC-544, respectively. The levels of CD22 and CD55 decreased 12-24 h after CMC-544 exposure, whereas those of CD20 increased. The levels of CD59 did not change significantly. (b) Results are shown from four representative patients with BCM that contained a sufficient number of cells for analysis. The same results were obtained for the remaining samples. 